\title{
Portuguese Adaptation of a Faux Pas Test and a Theory of Mind Picture Stories Task
}

\author{
Luís Faísca $^{a}$ Sofia Afonseca ${ }^{a} \quad$ Martin Brüne $^{b} \quad$ Gabriela Gonçalves $^{a}$ \\ Alexandra Gomes $^{\mathrm{a}} \quad$ Ana Teresa Martins ${ }^{\mathrm{a}}$ \\ ${ }^{a}$ Department of Psychology and Center for Biomedical Research - CBMR, University of Algarve, Faro, Portugal; \\ ${ }^{b}$ Division of Cognitive Neuropsychiatry and Psychiatric Preventive Medicine, LWL University Hospital, Ruhr \\ University Bochum, Bochum, Germany
}

\section{Key Words}

Theory of Mind · Faux Pas Recognition Test · Theory of Mind Picture Stories Task

\section{Abstract}

Background: Developmental concepts of 'Theory of Mind' (ToM) suggest that this cognitive domain is an innate human capacity requiring input from the social environment to mature. Research suggests substantial individual differences in ToM, depending on childhood experiences, genetics, and the presence or absence of a neuropsychiatric disorder. None of the existing ToM tests for adult populations have been made available in Portuguese. Accordingly, the main objective of this study was to evaluate the psychometric properties of the Portuguese versions of the Faux Pas (FP) Recognition Test and the ToM Picture Stories Task. Sampling and Methods: Both tests were given to a sample of 200 Portuguese adults (125 women and 75 men) aged between 18 and 60 years. Results and Conclusions: The psychometric analyses of the results indicate that the Portuguese version of the FP Recognition Test is appropriate for use in research and clinical settings, providing a com- posite score that reliably measures the ability to infer the thoughts and feelings of others in a 'faux pas' situation (Cronbach's $a=0.82$ ). However, the ToM Picture Stories Task did not show acceptable psychometric qualities.

(c) 2016 S. Karger AG, Basel

\section{Introduction}

Human interaction relies heavily on social cognition, comprising cognitive processes that allow people to think about themselves and others in the social world [1]. The ability to perceive, interpret, and explain the mental states and emotions of other persons is a crucial element of social cognition and has been denominated Theory of Mind (ToM), a term first used in 1978 by Premack and Woodruff [2], who suggested that chimpanzees, just like humans, could attribute mental states to others. ToM encompasses two main components: a social-perceptive and a social-cognitive process [3-5]. The social-perceptive component supports the ability to decode the mental states of others based on the observable and available data (perception of social cues), whilst the social-cognitive

\section{KARGER}

E-Mail karger@karger.com www.karger.com/psp (c) 2016 S. Karger AG, Basel

0254-4962/16/0493-0143\$39.50/0
Ana Teresa Martins, $\mathrm{PhD}$

Department of Psychology and Center for Biomedical Research - CBMR University of Algarve Campus de Gambelas PT-8005-139 Faro (Portugal)

E-Mail atmartins@ualg.pt 
component refers to the ability to reason about mental states in order to infer intentions and beliefs and to explain or predict the actions of others [5]. A broad array of tests has been developed to assess ToM processes, using different types of materials from simple presentations of socially relevant stimuli to live social interactions [6]. This range of methods includes the use of static schematic faces or photographs of faces, static images or photographs of people in complex social scenes, stories describing social interactions or dynamic images of people in interaction, and even real social interactions [7]. The tasks designed to evaluate the social-perceptive processes required by ToM mainly evoke the ability to infer emotions from the gaze cues, facial expressions, or vocal cues. For example, the 'Eyes Test' [8], originally named 'Reading the Mind in the Eyes Task', assesses the ability to decode the feelings and thoughts of others from the information conveyed by the upper face expression. The social-cognitive component of ToM involves the ability to use and integrate information from shared world knowledge and physical actions in order to infer the mental states of others and to predict their behavior. To assess this component, researchers usually present participants with short stories or pictures depicting social scenarios where two or more characters interact and ask participants to infer the mental states of the characters involved or to predict their behavior based on these inferences $[9,10]$. The Faux Pas (FP) Recognition Test [11] is one example of this type of task, assessing the ability to infer the thoughts and feelings of others in a 'faux pas' situation (a declaration or action that accidentally offends another person). Another task designed to assess the social-cognitive component is the ToM Picture Stories Task [12]. The principal objective of this test is to examine the ability to infer mental states and predict behaviors of others through the interpretation of the actions of cartoon characters. This kind of tasks has been largely used to investigate the social-perceptive and the social-cognitive components of ToM, both in clinical and nonclinical groups. Specifically, the FP test has frequently been used in different adult clinical groups, including alcohol dependence [13], brain lesions [11, 14], schizophrenia [15, 16], Parkinson's disease [17], and Alzheimer's dementia [18]. Similarly, the ToM Picture Stories task has been applied in the study of affective disorders such as schizophrenia [12, 19] and bipolar disorder [20, 21], Huntington's disease [22], and Borderline Personality Disorder [23]. The evaluation of ToM utilizing this kind of task has proved to be very useful, and the results demonstrate that they provide a sensitive measurement of the social-cognitive component of ToM, at least in severe mental disorders.
The theoretical relevance of the concept of ToM and its applicability to clinical and nonclinical settings renders necessary the existence of reliable and valid procedures to assess these abilities. However, only a few studies evaluated the psychometric properties of the ToM tasks, especially those which assess the social-cognitive component. For example, some authors presented psychometric data on the Eyes Test, the FP Test, and the Dewey Social Stories and obtained excellent reliability for the FP Test in a sample of Swedish adults [24]. In relation to the ToM Picture Stories Task, Bechi et al. [25] found adequate reliability indices in a clinical sample (patients with schizophrenia) and in a large representative population sample [26]. To our knowledge, there is no standardized test available in European Portuguese to assess the social component of ToM.

Given this rationale, our main objective was to study the psychometric properties of the Portuguese versions of the FP Recognition Test and the ToM Picture Stories Task in a sample of healthy Portuguese adult subjects.

\section{Methods}

\section{Participants}

A total of 200 individuals ( 125 women and 75 men) aged between 18 and 60 years $($ mean $=33.0 \pm 12.0)$ participated in the study. Concerning education level, $8 \%$ of the participants completed 4 th grade, $24 \%$ completed 9 th grade, $39 \%$ completed 12 th grade, and 29\% held a higher degree (postgraduation, bachelor degree, master degree, or $\mathrm{PhD}$ ). Regarding their place of origin, $34 \%$ of the participants were born in the south of Portugal, $27 \%$ in the center, $6.5 \%$ in the north, $25 \%$ on Madeira Island, and $7.5 \%$ in other Portuguese-speaking countries. Participants were recruited from the general population through informal invitation (personal contacts and social networks). The study protocol was previously approved by the Scientific Committee of the Faculty of Social and Human Sciences (University of Algarve).

\section{Instruments}

The FP Recognition Test evaluates the ability to infer the thoughts and feelings of others in a 'faux pas' situation. The test was roughly based on a previous version of the task of Baron-Cohen et al. [27] and was adapted to adults. This instrument comprises 20 stories ( 10 control stories and $10 \mathrm{FP}$ stories). The administration procedure was conducted according to the original instructions of Stone et al. [11]. Concerning the scoring instructions of Stone et al. [11] and Söderstrand and Almkvist [24], two scores were calculated separately for the FP Recognition Test. The FP score expresses the correctness on the questions about the $10 \mathrm{FP}$ stories. Participants scored 1 point for each correct answer to the six questions posed after each FP story; this global score ranged from 0 points (no correct answers) to 60 points (all questions answered correctly). A second score uses the first question of the control stories ( 2 points were given to each correct answer); this 
Table 1. Item analysis for the FP Recognition Test: accuracy (number and percentage of correct responses and above-chance probability for the FP and control stories) and number of correct FP questions (mean \pm SD and quartiles for the FP stories)

\begin{tabular}{|c|c|c|c|c|c|c|c|c|c|}
\hline \multicolumn{6}{|c|}{ FP stories } & \multicolumn{4}{|c|}{ Control stories } \\
\hline \multirow[t]{2}{*}{ items } & \multicolumn{3}{|c|}{ 'faux pas' detection } & \multicolumn{2}{|c|}{ FP questions } & \multirow[t]{2}{*}{ items } & \multicolumn{3}{|c|}{ 'faux pas' rejections } \\
\hline & $\mathrm{n}$ & $\%$ & $\mathrm{p}^{1}$ & mean $\pm S D$ & quartiles & & $\mathrm{n}$ & $\%$ & $\mathrm{p}^{1}$ \\
\hline 2 & 173 & 86.5 & 0.000 & $3.92 \pm 1.00$ & $3-4-5$ & 1 & 183 & 91.5 & 0.000 \\
\hline 4 & 156 & 78.0 & 0.000 & $2.85 \pm 0.83$ & $2-3-3$ & 3 & 193 & 96.5 & 0.000 \\
\hline 7 & 126 & 63.0 & 0.000 & $3.74 \pm 0.83$ & $3-4-4$ & 5 & 185 & 92.5 & 0.000 \\
\hline 11 & 149 & 74.5 & 0.000 & $3.41 \pm 0.86$ & $3-3-4$ & 6 & 171 & 85.5 & 0.000 \\
\hline 12 & 141 & 70.5 & 0.000 & $3.39 \pm 0.91$ & $3-3-4$ & 8 & 189 & 94.5 & 0.000 \\
\hline 13 & 86 & 43.0 & 0.056 & $3.28 \pm 1.08$ & $3-3-4$ & 9 & 172 & 86.0 & 0.000 \\
\hline 14 & 151 & 75.5 & 0.000 & $3.93 \pm 0.86$ & $3-4-5$ & 10 & 194 & 97.0 & 0.000 \\
\hline 15 & 140 & 70.0 & 0.000 & $2.85 \pm 0.97$ & $2-3-4$ & 17 & 194 & 97.0 & 0.000 \\
\hline 16 & 119 & 59.5 & 0.009 & $3.48 \pm 1.10$ & $3-4-4$ & 19 & 194 & 97.0 & 0.000 \\
\hline 18 & 108 & 54.0 & 0.289 & $3.30 \pm 0.90$ & $3-3-4$ & 20 & 176 & 88.0 & 0.000 \\
\hline
\end{tabular}

FP questions: number of correct answers to the FP questions after detecting the 'faux pas' situation.

${ }^{1}$ Binomial test for proportion $=0.5$.

second score, named the FP rejection score (FP-rej), ranges from 0 to 20 points and reflects the correct rejection of stories without 'faux pas' situations. In addition, we computed a third score, based exclusively on the first question of the FP stories (range: 0-10 points) and expressing just the correct identification of a 'faux pas' situation in these stories, the FP detection score (FP-det). If participants gave errors in the control questions, we assumed that the interpretation of the story was impaired. In these cases, and according to the suggestion of Stone et al. [11], the scores for these specific stories were deducted from the total score. The answer time (in seconds) spent in the task was also measured using a chronometer.

The ToM Picture Stories Task [12] assesses the ability to infer the mental states of others and to predict their behavior. In its original version it comprises 6 cartoon stories (each comprising four cards). The researcher present the four cards in random order and the participant is required to sort them in the correct sequence; 2 points are given to the first and last cards and 1 point to the middle cards in the right position. In case the sequence is incorrect, the researcher puts the cards in the right order and continues the task. Participants are then asked about the story, regarding true or false beliefs of the characters and the identification of actions of cooperation, reciprocity, deception, and fraud, thus testing the ability to infer the mental states of the characters in the story; 1 point is given to each correct answer and 0 points to incorrect answers. The total score of the task (adding both the scoring for the cards sequences and for the ToM questions) has a maximum of 59 points. Similarly to the FP task, the total answer time (in seconds) was also measured for the ToM stories task. This instrument has good internal consistency (Cronbach's $\alpha=0.85$ ) according to one study in outpatients affected by schizophrenia [25] and to an adaptation study for a recent computerized version of the test [26].

Portuguese Versions of the Faux Pas Test and the ToM Picture Stories Task

\section{Procedure}

Instrument Adaptation

The first step of this study comprised the translation of the FP Recognition Test and the ToM Picture Stories Task into European Portuguese by two independent experts in psychology with European Portuguese as their native language. Following the recommendations of Brislin [28], after reaching a consensus, the translated version was back-translated into English by a bilingual expert. Finally, a pretest was performed with 2 participants in order to correct any possible problems they had in understanding or completing the tasks.

\section{Data Collection}

The participants were selected using a nonprobabilistic sampling technique (nonintentional convenience sampling). The researcher approached and asked possible participants about their availability to participate in the study. The participants were informed about the aims of the study and the confidentiality of the data. They were required to read and sign an informed consent form to ensure that their participation was voluntary and conscious. In the next phase, the participants completed a sociodemographic questionnaire. They were then required to complete the FP Recognition Test and the ToM Picture Stories Task in order to evaluate their ability in deducing the intentions, emotions, and beliefs of others. The tests were administrated always in the same sequence. The participants were evaluated in an isolated and noiseless room in two university campuses.

\section{Data Analysis}

The data were processed with the IBM SPSS software. The psychometric analysis comprised item analysis, exploratory factor analysis, and reliability and validity analysis. Considering the dichotomous nature of some of the items, when necessary we factor 
analyzed the tetrachoric correlation matrix using the unweighted least squares (ULS) procedure, as recommended by some authors [29]; tetrachoric correlations were estimated using the TETRACOM script [30]. For internal consistency analysis, Cronbach's a and respective bootstrapped 95\% confidence intervals (95\% CI) were computed (using 2,000 samples). A significance level of 0.05 was considered in all inferential statistical tests.

\section{Results}

\section{Psychometric Properties of the Two Social-Cognitive}

Tasks

FP Recognition Test

Item Analysis. Table 1 presents the percentages of correct 'faux pas' detections for the $10 \mathrm{FP}$ stories and the percentages of correct 'faux pas' rejections for the 10 control stories. Correct rejection percentages were typically higher (85.5-97.0\%) than correct 'faux pas' detection percentages (43.0-86.5\%). For the FP stories 13 and 18, the percentage of correct detections is not reliably above chance; however, the mean number of correctly answered FP questions (an average of 3.3 correct answers out of the five FP questions) suggests that the participants who answered 'yes' truly understood the 'faux pas' situation depicted in those particular stories. Considering the number of correct FP questions after a positive answer to the FP stories, the median was $\geq 3$, indicating that at least $50 \%$ of the participants who detected the 'faux pas' situation have answered correctly at least to three out of the five FP questions used in each story. Overall, these results suggest that while most participants easily identify the absence of 'faux pas' situations in all control stories, the detection of such situations entails more difficulties.

Exploratory Factor Analysis. Exploratory factor analyses were performed to assess the dimensionality of the FP Recognition Test. We analyzed separately the 10 FP stories and the 10 control Stories, considering the differences in the response pattern for these two types of items.

For the FP stories, a tetrachoric correlation matrix was computed for the answers to the 'faux pas' detection question (first FP question). The Kaiser-Meyer-Olkin (KMO) index and Bartlett's sphericity test showed the appropriateness of this correlation matrix for factor analysis $\left(\mathrm{KMO}=0.810, \chi^{2}(45)=1,154.5, \mathrm{p} \leq 0.001\right)$. After applying a common variance extraction method (ULS), a single-factor solution emerged: the first eigenvalue of the reduced correlation matrix was 4.910. For this one-factor solution, all loadings were greater than 0.6 (0.617-0.809), except for item 2 , which had a loading of 0.556 . The alternative two-factor structure included a second factor with
Table 2. Factor analysis of the FP Recognition Test: one-factor solution for the FP detections, FP score, and FP rejections

\begin{tabular}{|c|c|c|c|c|}
\hline \multicolumn{3}{|l|}{ FP stories } & \multicolumn{2}{|l|}{ Control stories } \\
\hline items & $\begin{array}{l}\mathrm{FP} \\
\text { detection }\end{array}$ & FP score & items & $\begin{array}{l}\mathrm{FP} \\
\text { rejection }\end{array}$ \\
\hline 2 & 0.556 & 0.443 & 1 & 0.385 \\
\hline 4 & 0.683 & 0.508 & 3 & 0.740 \\
\hline 7 & 0.617 & 0.485 & 5 & 0.262 \\
\hline 11 & 0.800 & 0.620 & 6 & 0.003 \\
\hline 12 & 0.660 & 0.579 & 8 & 0.098 \\
\hline 13 & 0.691 & 0.555 & 9 & 0.206 \\
\hline 14 & 0.809 & 0.607 & 10 & 0.181 \\
\hline 15 & 0.692 & 0.630 & 17 & 0.745 \\
\hline 16 & 0.775 & 0.663 & 19 & 0.823 \\
\hline 18 & 0.683 & 0.589 & 20 & 0.189 \\
\hline Eigenvalues & 4.910 & 3.268 & Eigenvalues & 2.118 \\
\hline$\% \mathrm{EV}$ & 55.4 & 39.3 & $\% \mathrm{EV}$ & 26.2 \\
\hline RMSR & 0.080 & 0.053 & RMSR & 0.116 \\
\hline Cronbach's a & 0.816 & 0.825 & Cronbach's a & 0.568 \\
\hline
\end{tabular}

Eigenvalues: eigenvalues of the reduced correlation matrix. $\% \mathrm{EV}$ : explained percentage of total variance based on the eigenvalues of the original correlation matrix. RMSR: for a good model, RMSR $<0.07$.

a low eigenvalue (0.712); this second factor was preferably associated with items 4 and 2, but factor loadings were low (loading for item $2=0.462$ ), and several cross-loadings ( $>0.4)$ made its interpretation difficult. In addition, a parallel analysis [31] suggested that the two-factor solution was not appropriated to this data set. Thus, the onefactor solution seems to be more adequate. The root mean square of residuals (RMSR) index (0.080) suggested that this one-factor solution predicts reasonably well the original correlation matrix, although story 02 seems to be less well represented (communality $=0.309$ ). Table 2 summarizes the factor analysis of the answers to the first FP question.

Considering the FP scores (the sum of points obtained for the six questions in each FP story), all 10 items showed signs of bimodality due to the presence of a high number of zeros resulting from the selective nature of the first FP question (that is, an FP score of 0 occurred always when the first FP question was negative). Despite the nonnormal distributions of those scores, a second exploratory factor analysis was done on the 10 items, this time using the Pearson correlation matrix. The KMO index and Bartlett's sphericity test showed the appropriateness of the correlation matrix for the factor analysis $(\mathrm{KMO}=$ 
Table 3. Descriptive statistics and normality tests for the FP test composite scores

\begin{tabular}{lccccccc}
\hline & Mean \pm SD & Range & Quartiles & Skewness & Kurtosis & S-W & $p$ \\
\hline FP-det & $6.74 \pm 2.79$ & $0-10$ & $5-7.5-9$ & -0.80 & -0.17 & 0.905 & 0.000 \\
FP score & $29.86 \pm 13.35$ & $0-54$ & $21-32-40$ & -0.48 & -0.50 & 0.964 & 0.000 \\
FP-rej & $18.51 \pm 2.35$ & $4-20$ & $18-20-20$ & -2.73 & 10.75 & 0.651 & 0.000 \\
\hline
\end{tabular}

FP-det: composite measure for the correct detection of 'faux pas' situations (range 0-10). FP score: composite measure for the correctness of the question about the 'faux pas' situations (range 0-60). FP-rej: composite measure for the correct rejection of non-'faux pas' situations (range $0-20$ ). S-W = Shapiro-Wilk normality test statistic.

$\left.0.867, \chi^{2}(45)=488.2, \mathrm{p} \leq 0.001\right)$. After applying the ULS factor extraction method, a single-factor solution emerged (first eigenvalue of the reduced correlation matrix = 3.268). For this one-factor solution, all of the factor loadings were greater than $0.5(0.508-0.663)$, except for items 7 and 2 (loadings of 0.485 and 0.443 , respectively). The RMSR value was adequate (0.053). The alternative twofactor structure included a second factor with an eigenvalue smaller than one (0.803) and exclusively associated with item 2. Furthermore, a Horn's parallel analysis did not support the two-factor solution. Thus, one-factor solution seems adequate to represent the variability of the answers to the FP questions. The loadings for the onefactor solution are presented in table 2.

The tetrachoric correlation matrix for the control stories was a nonpositive definite matrix and had to be smoothed before analysis [30]. The appropriateness of this smoothed correlation matrix was modest (KMO index $=0.630$; Bartlett's sphericity test $\chi^{2}(45)=268.5$, $\mathrm{p} \leq$ $0.001)$. Using a common variance extraction method (ULS), a single-factor solution emerged (eigenvalue = 2.118). Only 3 items had relevant loadings in this factor, namely stories 03 (0.740), 17 (0.745) and 19 (0.823); all others had loadings $<0.4$, a result that seems to be a consequence of the smoothing procedure. The RMSR index suggests that the one-factor solution is not reasonable (0.116; the largest fitted correlation residual was 0.36 , indicating a clear poor fit). However, the alternative twofactor structure was not supported by a parallel analysis. Table 2 summarizes the one-factor solution for the answers to the control stories.

Reliability Analysis. Internal consistency, as measured by Cronbach's a, was 0.82 for correct FP-det (bootstrapped 95\% CI: 0.76-0.85), 0.82 for the FP question score (bootstrapped 95\% CI: 0.78-0.86) and 0.57 for FPrej (bootstrapped 95\% CI: 0.31-0.71). Only composite scores based on FP stories have acceptable internal reliabilities, suggesting that the FP test has a better performance in the 'faux pas' identification measure than discriminating the correct rejection of 'faux pas'.

Composite Scores. FP detection and correct rejection scores, as well as FP total score, were calculated according to the scoring instructions. Table 3 presents the descriptive results for these composite scores.

The mean score for correct FP detection (FP-det) was 6.74 , corresponding to an average accuracy percentage of $67.4 \%$, while the mean score for correct rejection of a 'faux pas' situation (FP-rej) was 18.51 (average accuracy percentage: 92.6\%). Ceiling effects occurred for both measures but were particularly evident for the correct rejection score (86.0\% of participants reached a ceiling for FP-rej vs. only $17.5 \%$ for FP-det). Concerning the FP score, the mean corresponds to an accuracy percentage of $49.8 \%$, and the participants failed to answer correctly all the questions about the 'faux pas' scenarios (the maximum score obtained was of 54 points, with a median of 32 out of 60 points).

All three scores failed the Shapiro-Wilk normality test; although distributions were asymmetric (negative skewness), only FP-rej seems to be markedly left skewed and peaked (due to the presence of a ceiling effect), demonstrating the low sensitivity of this measure.

The correlation between the three scores reveals, as expected, that FP detection and FP score are strongly correlated $(\mathrm{r}=0.96, \mathrm{p} \leq 0.001)$; however, both scores are weakly and negatively correlated with FP-rej (FP-det: $r=$ $-0.19, \mathrm{p}=0.007$; FP score: $\mathrm{r}=-0.15, \mathrm{p}=0.034)$. These results suggest that participants evaluated the control stories somehow differently from the FP stories.

There were gender significant differences both in FP$\operatorname{det}($ Cohen's $d=0.32, t=2.15, p=0.034)$ and in FP questions score (Cohen's $\mathrm{d}=0.34, \mathrm{t}=2.37, \mathrm{p}=0.019$ ), with 
Table 4. Item analysis for the ToM Picture Stories Task: number and percentage of correct sequence responses and questionnaire accuracy

\begin{tabular}{llllll}
\hline \multirow{2}{*}{ Items } & \multicolumn{2}{l}{ Correct sequence } & & \multicolumn{2}{c}{ Questionnaire accuracy } \\
\cline { 2 - 3 } \cline { 5 - 6 } & $\mathrm{n}$ & $\%$ & & mean \pm SD & $\%$ \\
\hline 1 & 150 & 75.0 & & $1.97 \pm 0.19$ & 98.5 \\
2 & 127 & 63.5 & & $4.45 \pm 0.92$ & 88.9 \\
3 & 190 & 95.0 & & $3.66 \pm 0.71$ & 91.4 \\
4 & 192 & 96.5 & & $1.99 \pm 0.10$ & 99.5 \\
5 & 158 & 79.0 & & $4.72 \pm 0.52$ & 94.4 \\
6 & 133 & 66.5 & & $4.65 \pm 0.63$ & 93.0 \\
\hline
\end{tabular}

Questionnaire accuracy: maximum score for questions is 2 for stories 1 and 4, 4 for story 3 , and 5 for stories 2, 5, and 6 .

female participants showing a better performance (see table 4). Gender did not influence the FP-rej (Cohen's $\mathrm{d}=0.18, \mathrm{t}=1.26, \mathrm{p}=0.208)$. The three scores were not significantly affected by age (FP-det: $\mathrm{r}=-0.09$, nonsignificant; FP score: $r=-0.13, p=0.078$; FP-rej: $r=-0.03$, nonsignificant) but were mildly affected by education level (FP-det: $\mathrm{F}_{2,197}=4.67, \mathrm{p}=0.010, \eta^{2}=0.046$; FP score: $\mathrm{F}_{2,197}=4.15, \mathrm{p}=0.017, \eta^{2}=0.040 ; \mathrm{FP}-$ rej: $\mathrm{F}_{2,197}=2.07$, $\left.\mathrm{p}=0.128, \eta^{2}=0.021\right)$. The participants who completed elementary school obtained significantly lower results on FP detection and FP score than the participants with higher education levels (Tukey's HSD test, $\mathrm{p}<0.05$ ).

Finally, from a convergent validity perspective, we correlated the three FP scores with the global score from the ToM Picture Stories Task. The obtained correlations were positive and small for FP stories (FP-det: $\mathrm{r}=0.18$, $\mathrm{p}=0.013$; FP score: $\mathrm{r}=0.16, \mathrm{p}=0.021)$ but nonsignificant for control stories (FP-rej: $\mathrm{r}=-0.10, \mathrm{p}=0.145$ ).

ToM Picture Stories Task

Item Analysis. Table 4 presents the percentages of correct sequences for each of the 6 stories in the ToM Picture Stories Task; performance was particularly high for stories 3 and 4 (above 95\%) and clearly worse for stories 2 and 6 (below 70\%). However, the mean score for the subsequent questions was systematically high (accuracy scores corresponding to percentages always above $90 \%$, except for story 2). Overall, the results suggest a clear comprehension of all the stories, even if the initial sequencing of the pictures was not perfect.

Internal Consistency. Internal consistency measures were calculated for three composite measures: the sequencing score (Cronbach's $\alpha=0.51$; bootstrapped $95 \%$
CI: 0.29-0.65), the ToM questionnaire score (Cronbach's $\alpha=0.47$; bootstrapped 95\% CI: $0.32-0.59$ ), and the overall ToM Picture Stories Task score (Cronbach's $\alpha=0.54$; bootstrapped 95\% CI: 0.33-0.67). Although these unsatisfactory reliability indices may be attributed to the small number of items of the test ( 6 items), the corrected itemtotal correlations and the correlation between items remained in the low range (median $\mathrm{r}<0.28$ and median $\mathrm{r}<$ 0.18 , respectively), suggesting the weak internal consistency of the results on this task.

Composite Scores. Several measures were calculated following the scoring instructions [12]: comprehension of first- and second-order beliefs, comprehension of false first-, second- and third-order beliefs, reality measure, and comprehension of reciprocity, deception, and cheating detection. As previously stated, three global scores for the ToM Picture Stories Task were also calculated: sequencing, questionnaire, and overall score (table 5).

Overall, the participants performed well on the ToM Picture Stories Task, with a mean of 52.76 and a median of 54 points out of $59 ; 15 \%$ of the sample reached the maximum score (table 6). The participants seemed to perform poorly on the comprehension of false third-order beliefs (mean $=2.41$ out of a maximum of 3 points; ceiling performance $=57.5 \%)$. No differences were observed for gender $(p>0.1$, Cohen's $d<0.22)$ in all composite measures. Age seemed to negatively affect the comprehension of first-order beliefs $(r=-0.31, p \leq 0.001)$, the comprehension of second-order beliefs $(\mathrm{r}=-0.16, \mathrm{p}=$ 0.027 ), and the comprehension of third-order false beliefs $(\mathrm{r}=-0.22, \mathrm{p}=0.002)$, resulting in a significant negative effect both for the questionnaire score $(\mathrm{r}=-0.28$, $\mathrm{p} \leq$ $0.001)$ and for the overall ToM Picture Stories Task ( $\mathrm{r}=$ $-0.17, \mathrm{p}=0.018$ ). Education level seemed to affect significantly only the comprehension of second-order false beliefs $\left(\mathrm{F}_{2,197}=2.9, \mathrm{p}=0.057, \eta^{2}=0.029\right)$; participants who completed the elementary school obtained significantly lower results than participants with higher education levels (means: 2.74 vs. 2.83; Tukey's HSD test, $\mathrm{p}<$ 0.05).

\section{Discussion}

Social-cognitive skills have been a major focus of research in both clinical and nonclinical samples [32-34]. Specifically concerning ToM, deficits have been linked to poor social functioning in several clinical populations [35-37]. 
Table 5. Descriptive statistics of the ToM Picture Stories Task measures

\begin{tabular}{|c|c|c|c|c|}
\hline & Range & Mean \pm SD & $\begin{array}{l}\text { Mean } \\
\text { accuracy, } \\
\%\end{array}$ & $\begin{array}{l}\text { Participants } \\
\text { reaching } \\
\text { maximum } \\
\text { score, \% }\end{array}$ \\
\hline Comprehension of first-order beliefs ( $\max 5$ ) & $3-5$ & $4.82 \pm 0.48$ & 96.4 & 86.0 \\
\hline First-order belief $(\max 2)$ & $0-2$ & $1.87 \pm 0.39$ & 96.5 & 89.0 \\
\hline False first-order belief $(\max 3)$ & $2-3$ & $2.95 \pm 0.22$ & 98.3 & 95.0 \\
\hline Comprehension of second-order beliefs ( $\max 5)$ & $1-5$ & $4.68 \pm 0.62$ & 93.6 & 74.0 \\
\hline Second-order belief (max 2) & $1-2$ & $1.98 \pm 0.16$ & 99.0 & 97.5 \\
\hline False second-order belief ( $\max 3)$ & $0-3$ & $2.70 \pm 0.58$ & 90.0 & 75.5 \\
\hline Comprehension of false third-order beliefs $(\max 3)$ & $0-3$ & $2.41 \pm 0.79$ & 80.3 & 57.5 \\
\hline Reality questions ( $\max 2)$ & $1-2$ & $1.99 \pm 0.10$ & 99.5 & 99.0 \\
\hline Comprehension of reciprocity $(\max 3)$ & $1-3$ & $2.93 \pm 0.30$ & 97.7 & 93.5 \\
\hline Comprehension of deception $(\max 3)$ & $1-3$ & $2.84 \pm 0.38$ & 94.7 & 84.5 \\
\hline Comprehension of cheating detection $(\max 2)$ & $0-2$ & $1.90 \pm 0.35$ & 95.0 & 91.0 \\
\hline Sequencing score $(\max 36)$ & $3-36$ & $31.20 \pm 5.12$ & 86.7 & 31.0 \\
\hline Questionnaire score $(\max 23)$ & $13-23$ & $21.55 \pm 1.83$ & 93.7 & 43.5 \\
\hline Overall measure ( $\max 59)$ & $22-59$ & $52.76 \pm 5.80$ & 89.4 & 15.0 \\
\hline
\end{tabular}

Table 6. Descriptive statistics (mean \pm SD) for FP scores by gender and education level

\begin{tabular}{llll}
\hline & FP-det & FP score & FP-rej \\
\hline Gender & & & \\
$\quad$ Male $(\mathrm{n}=75)$ & $6.17 \pm 3.08$ & $27.00 \pm 14.01$ & $18.24 \pm 2.87$ \\
$\quad$ Female $(\mathrm{n}=125)$ & $7.08 \pm 2.55$ & $31.57 \pm 12.69$ & $18.67 \pm 1.97$ \\
\hline Educational level & & & \\
$\quad$ Elementary school grades 4 and 9 $(\mathrm{n}=64)$ & $5.89 \pm 3.06$ & $25.98 \pm 14.15$ & $19.00 \pm 2.36$ \\
$\quad$ High school $(\mathrm{n}=78)$ & $7.03 \pm 2.74$ & $31.32 \pm 13.45$ & $18.28 \pm 4.27$ \\
$\quad$ College $(\mathrm{n}=58)$ & $7.29 \pm 2.32$ & $32.15 \pm 11.47$ & $18.27 \pm 2.38$ \\
\hline
\end{tabular}

However, there is a lack of psychometrically validated standardized instruments to assess ToM competence in adult populations. The clinical usefulness of these tasks depends heavily on the adaptation of the experimental procedures, as well as their validity and reliability. Recently, an effort has been made to develop standardized procedures, with good psychometric proprieties, to assess ToM competence both in adults and in children [24, 38, 39]. We believe that making these kinds of instruments available is particularly useful in both research and clinical settings, since they allow assessing the ability to infer the mental states of others and help in monitoring the development of patients' social-cognitive skills during therapy.

The main goal of this study was to evaluate the psychometric qualities of the Portuguese version of two tools measuring the social-cognitive component of ToM ability in adults, namely the FP Recognition Test and the ToM Picture Stories Task.

The Portuguese version of the FP Recognition Test had good psychometric properties. The scores based either on the first FP question (FP-det) or on the total FP questions (FP score) provided a reliable one-dimensional measure of the ability to infer the thoughts and feelings of others in a 'faux pas' situation (Cronbach's $\alpha>0.8$ ). Conversely, the measure based on the correct rejection of absent 'faux pas' situations (control stories) correlated negatively with the previous measures and seemed not to have reasonable psychometric properties; the participants were good at rejecting the situations where no 'faux pas' occurred, resulting in a near-ceiling performance (accuracy close to $90 \%$ ) and, consequently, in a low vari- 
ability of the data with low sensitivity and reliability (Cronbach's $\alpha<0.6$ ). These findings suggest that the sensitivity of the FP Recognition Test is higher for the detection of 'faux pas' situations than for the correct rejection of non-'faux pas' situations. Therefore, the control stories may be considered filler items and should not be used as a valid score, at least for healthy individuals. However, we may consider that the inability to correctly reject non'faux pas' situations may indicate an impairment in ToM that can be useful for the assessment of clinical groups.

Several measures can be calculated according to the scoring instructions of the ToM Picture Stories Task, although internal consistency indices were computed just for the overall measure, the sequencing score, and the questionnaire score. This instrument seems to have a poor reliability both for the sequence and overall measures (Cronbach's $a<0.6$ ), showing a better, but still unsatisfactory internal consistency for the ToM questionnaire score $(\alpha=0.65)$. The procedure for scoring the sequences may have contributed to these low reliability indices; indeed, at least 2 stories were not perfectly sorted by a third of the sample, but this did not affect the comprehension of these stories, as expressed by high scores on the questionnaire component. Perhaps alternative sequences of pictures are compatible with the content of the stories, and this possibility should be considered in the scoring procedure. The low reliability observed for the Portuguese version of the ToM Picture Stories Task is somewhat surprising, as two recent studies showed adequate reliability scores in an Italian clinical sample [25] and in a recent computerized adaptation of the ToM Picture Stories Task with a large representative population sample [26]. As in the Portuguese version, Cronbach's $\alpha$ values were better for the questionnaire part compared to the sequencing part. However, these validation studies included 12 instead of 6 cartoon stories, which may have improved the psychometric properties of the task. Furthermore, considering that in the present study the instrument was applied after the FP Recognition Test, the results of the ToM Picture Stories Task may have been biased by internal stimuli affecting the cognitive resources required by the task (e.g. decreased motivation caused by the slowness of the task or decreased perception and attention during the task performance); however, the questionnaire score was systematically high for all 6 items (accuracy $\geq 89 \%$ ), suggesting that the participants comprehended the stories. Other possible explanations may rely on a cultural adaptation problem, worsened by the subjective concepts and heterogeneous content included in the instrument.
The effect of sociodemographic characteristics of the participants on both tasks was analyzed. Women seem to be slightly better than men at the 'faux pas' identification (Cohen's $d=0.3$ ). However, gender differences were not found for the ToM Picture Stories Task. Women's superiority in performance with regard to empathy has repeatedly been described since the original study of BaronCohen and Wheelwright [40]. For example, Bosacki [41] observed that female preadolescents were better than their male counterparts on the intrapersonal task of constructing a coherent sense of self and on the interpersonal task of understanding multiple and contradictory intentions of others. More recently, other authors also verified that women performed better in the FP test than men [42]. Hence, our results seem to support the sensibility of the FP Recognition Test (namely, the FP-det) to discriminate female superiority in recognizing and inferring mental states and predicting behavior in others.

Education level had a positive effect on the detection of 'faux pas' situations (FP Recognition Test) and on the comprehension of second-order belief scores (ToM Picture Stories Task). These were small nonsystematic effects, suggesting that the performance on those tasks is not much affected by education level.

The effects of age on the FP Recognition Test were negative but negligible. In contrast, age affected the performance on the ToM Picture Stories Task, namely the comprehension of first-, second-, and third-order beliefs, as well as the overall score; these effects were negative and small, but significant. Older individuals seemed to have more difficulties in identifying these beliefs in the stories presented, a relation also found in recent literature [43] and which continues to be a matter of debate since the process is not clear [44]. A meta-analysis of the influence of age on ToM task performance verified that older adults were found to perform more poorly than young adults, with deficits along all task types, domains, and modalities [43]. The reasons for this social reasoning decline with increasing age has been a matter of debate in the literature and seems not to be clearly linked with changes in other aspects of domain-general cognitive functioning, such as executive functioning [44].

The adaptation of the FP Recognition Test and the ToM Picture Stories Task to the Portuguese adult population has particular importance, not only because Portuguese is a widespread language but also because those instruments have strong clinical and scientific applicability. According to our findings, we suggest that the Portuguese version of the FP Recognition Test has good psychometric qualities and can be used with normal and clinical 
populations. On the contrary, the poor internal consistency observed for the measures provided by the ToM Picture Stories Task indicates that the Portuguese version of this instrument needs a more detailed investigation and improvement, and may only be suitable for adults with severe mental disorders. Notwithstanding the relevance of our results, we faced some limitations during the development of this study that did not allow us to draw more general conclusions from the data. Some aspects to highlight are the following: the duration of the tasks was perhaps too long, preventing the collection of a larger number of participants and perhaps affecting their motivation, and the sample was predominantly constituted by females and had a high percentage of students, which cre- ated a bias in relation to the sociodemographic characteristics of the Portuguese population. In order to minimize the effects of those biases, results were presented separately for gender and education level. Apart from these limitations, this study provides valuable insights about a field of increasing importance for clinical studies. Future research may benefit from using a larger sample, preferably with individuals with clinical disorders.

\section{Acknowledgment}

This work was partially supported by the FCT - Fundação para a Ciência e Tecnologia - through the Research Center grant UID/ BIM/04773/2013 CBMR 1334.

\section{References}

1 Beer JS, Ochsner KN: Social cognition: A multi-level analysis. Brain Res 2006;1079:98105.

2 Premack D, Woodruff G: Chimpanzee problem-solving: a test for comprehension. Science 1978;202:532-535.

-3 Tager-Flusberg H, Sullivan K: A componential view of theory of mind: evidence from Williams syndrome. Cognition 2000;76:5989.

4 Coricelli G: Two levels of mental states attribution: from automaticity to voluntariness. Neuropsychologia 2005;43:294-300.

5 Sabbagh MA, Moulson MC, Harkness KL: Neural correlates of mental state decoding in human adults: an event-related potential study. J Cogn Neurosci 2004;16:415-426.

-6 Risko EF, Laidlaw KEW, Freeth M, Foulsham $\mathrm{T}$, Kingstone A: Social attention with real versus reel stimuli: toward an empirical approach to concerns about ecological validity. Front Hum Neurosci 2012;6:1-11.

7 Byom LJ, Mutlu B: Theory of mind: mechanisms, methods, and new directions. Front Hum Neurosci 2013;7:1-12.

8 Baron-Cohen S, Wheelwright S, Hill J, Raste Y, Plumb I: The 'Reading the Mind in the Eyes' test revised version: a study with normal adults, and adults with Asperger syndrome or high-functioning autism. J Child Psychol Psychiatry 2001;42:241-251.

-9 Havet-Thomassin V, Allain P, EtcharryBouyx F, Le Gall D: What about theory of mind after severe brain injury? Brain Inj 2006; 20:83-91.

10 Happé FG: An advanced test of theory of mind: understanding of story characters' thoughts and feelings by able autistic, mentally handicapped, and normal children and adults. J Autism Dev Disord 1994;24:129-154.
11 Stone VE, Baron-Cohen S, Knight RT: Frontal lobe contributions to theory of mind. J Cogn Neurosci 1998;10:640-656.

12 Brüne M: Theory of mind and the role of IQ in chronic disorganized schizophrenia. Schizophr Res 2003;60:57-64.

13 Thoma P, Winter N, Juckel G, Roser P: Mental state decoding and mental state reasoning in recently detoxified alcohol-dependent individuals. Psychiatry Res 2013;205:232-240.

-14 Lee TM, Ip AK, Wang K, Xi C, Hu P, Mak HK, et al: Faux pas deficits in people with medial frontal lesions as related to impaired understanding of a speaker's mental state. Neuropsychologia 2010;48:1670-1676.

15 Abu-Akel A, Abushua'leh K: 'Theory of mind' in violent and nonviolent patients with paranoid schizophrenia. Schizophr Res 2004;69: 45-53.

16 Zhu C-Y, Lee TM, Li X-S, Jing S-C, Wang Y-G, Wang K: Impairments of social cues recognition and social functioning in Chinese people with schizophrenia. Psychiatry Clin Neurosci 2007;61:149-158.

17 Péron J, Vicente S, Leray E, Drapier S, Drapier D, Cohen R, et al: Are dopaminergic pathways involved in theory of mind? A study in Parkinson's disease. Neuropsychologia 2009;47:406-414.

-18 Gregory C, Lough S, Stone V, Erzinclioglu S, Martin L, Baron-Cohen S, et al: Theory of mind in patients with frontal variant frontotemporal dementia and Alzheimer's disease: Theoretical and practical implications. Brain 2002;125:752-764.

19 Brüne M, Bodenstein L: Proverb comprehension reconsidered - 'theory of mind' and the pragmatic use of language in schizophrenia. Schizophr Res 2005;75:233-239.
20 Barrera Á, Vázquez G, Tannenhaus L, Lolich M, Herbst L: Teoría de la mente y funcionalidad en pacientes bipolares en remisión sintomática. Rev Psiquiatr y Salud Ment 2013;6: 67-74.

21 Martino DJ, Strejilevich SA, Fassi G, Marengo E, Igoa A: Theory of mind and facial emotion recognition in euthymic bipolar I and bipolar II disorders. Psychiatry Res 2011;189:379_ 384.

22 Brüne M, Blank K, Witthaus H, Saft C: 'Theory of mind' is impaired in Huntington's Disease. Mov Disord 2011;26:671-678.

23 Ghiassi V, Dimaggio G, Brüne M: Dysfunctions in understanding other minds in borderline personality disorder: a study using cartoon picture stories. Psychother Res 2010; 20:657-667.

24 Söderstrand P, Almkvist O: Psychometric data on the Eyes Test, the Faux Pas Test, and the Dewey Social Stories Test in a populationbased Swedish adult sample. Nord Psychol 2012;64:30-43.

25 Bechi M, Riccaboni R, Ali S, Fresi F, Buonocore M, Bosia M, et al: Theory of mind and emotion processing training for patients with schizophrenia: preliminary findings. Psychiatry Res 2012;198:371-377.

26 Schuhfried: Wiener Testsystem. Test Manager Program. 2005.

27 Baron-Cohen S, O’Riordan M, Stone V, Jones $\mathrm{R}$, Plaisted K: Recognition of faux pas by normally developing children and children with Asperger syndrome or high-functioning autism. J Autism Dev Disord 1999;29:407-418.

28 Brislin RW: The wording and translation of research instruments; in Lonner WJ, Berry JW (eds): Field Methods in Cross-Cultural Research. Thousand Oaks, Sage Publications, 1986, pp 137-164. 
29 Knol DL, Berger MP: Empirical comparison between factor analysis and multidimensional item response models. Multivariate Behav Res 1991;26:457-477.

-30 Lorenzo-Seva U, Ferrando PJ: TETRA-COM: A comprehensive SPSS program for estimating the tetrachoric correlation. Behav Res Methods 2012;44:1191-1196.

-31 Horn JL: A rationale and test for the number of factors in factor analysis. Psychometrika 1965;30:179-185.

- 32 Bora E, Eryavuz A, Kayahan B, Sungu G, Veznedaroglu B: Social functioning, theory of mind and neurocognition in outpatients with schizophrenia; mental state decoding may be a better predictor of social functioning than mental state reasoning. Psychiatry Res 2006; 145:95-103.

33 Macrae CN, Bodenhausen GV: Social cognition: categorical person perception. Br J Psychol 2001;92:239-255.

34 Rutherford MD, Baron-Cohen S, Wheelwright $S$ : Reading the mind in the voice: a study with normal adults and adults with Asperger syndrome and high functioning autism. J Autism Dev Disord 2002;32:189-194.

35 Baron-Cohen S, Leslie AM, Frith U: Does the autistic child have a 'theory of mind'? Cognition 1985;21:37-46.

36 Brüne M, Brüne-Cohrs U: Theory of mind evolution, ontogeny, brain mechanisms and psychopathology. Neurosci Biobehav Rev 2006;30:437-455

37 Langdon R, Corner T, McLaren J, Ward PB, Coltheart M: Externalizing and personalizing biases in persecutory delusions: the relationship with poor insight and theory-of-mind. Behav Res Ther 2006;44:299-713.

38 Blijd-Hoogewys EM, van Geert PL, Serra M, Minderaa RB: Measuring theory of mind in children. Psychometric properties of the ToM Storybooks. J Autism Dev Disord 2008;38: 1907-1930.

39 Hutchins TL, Prelock PA, Bonazinga L: Psychometric evaluation of the Theory of Mind Inventory (ToMI): a study of typically devel- oping children and children with autism spectrum disorder. J Autism Dev Disord 2012;42: 327-341.

40 Baron-Cohen S, Wheelwright S: The empathy quotient: an investigation of adults with Asperger syndrome or high functioning autism, and normal sex differences. J Autism Dev Disord 2004;34:163-175.

41 Bosacki SL: Theory of mind and self-concept in preadolescents: links with gender and language. J Educ Psychol 2000;92:709-717.

42 Ahmed FS, Miller LS: Executive function mechanisms of theory of mind. J Autism Dev Disord 2011;41:667-678.

43 Henry JD, Phillips LH, Ruffman T, Bailey PE: A meta-analytic review of age differences in theory of mind. Psychol Aging 2013;28:826839.

44 Bernstein DM: Theory of mind through the ages: older and middle-aged adults exhibit more errors than do younger adults on a continuous false belief task. Exp Aging Res 2011; 37:481-502. 